\title{
Síntesis y caracterización de composites de TPOS reforzados con nanotubos de carbono
}

\author{
J. BIAGIOTTI', L. VALENTINI', J. M. KENNY'1', M. A. LÓPEZ MANCHADO²*, M. ARROYO² \\ ${ }^{1}$ Materials Engineering Center, Università di Perugia, 05100-Terni - Italy \\ ${ }^{2}$ Instituto de Ciencia y Tecnología de Polímeros, 28006-Madrid - España
}

\begin{abstract}
En el presente estudio se han preparado nanocomposites de elastómeros termoplásticos basados en mezclas de polipropileno (PP) y terpolímeros de etileno-propileno dieno (EPDM) reforzados con nanotubos de carbono. En concreto, se ha analizado el efecto de la incorporación de los nanotubos sobre las propiedades físicas y térmicas del material. Los resultados indican que los nanotubos actúan como nucleantes, acelerando notablemente la cristalización del PP en mezclas PP-EPDM. Es importante destacar que este efecto no es lineal con el porcentaje de nanotubos en el composite, observándose una saturación a altos contenidos de nanotubos. Se ha observado que la máxima nucleación se obtiene a concentraciones de $0.5 \%$ de nanotubos en el composite. El análisis mecanodinámico muestra resultados similares, observándose un aumento del módulo de almacenamiento a bajos contenidos de nanotubos, disminuyendo considerablemente a concentraciones de nanotubos superiores al $0.5 \%$. Por su parte, la espectroscopía Raman y microscopía electrónica de barrido permitieron determinar que estos cambios en las propiedades se deben a cambios estructurales en el composite.
\end{abstract}

Palabras claves: nanotubos de carbono, TPOs, espectroscopía Raman

\section{Synthesis and Characterization of Composites based on TPOs Reinforced with Carbon Nanotubes}

Polymer composites based on thermoplastic elastomer and single-walled carbon nanotubes have been investigated. In particular, the effect of the incorporation of SWNTs on physical and thermal properties of the material are described. The results indicate that the SWNT act as nucleant agent, sensibly accelerating the PP crystallization in PP-EPDM blends. It is of interest to note that this effect is not linearly dependent on the SWNTs' content, showing a saturation of the nucleant effect at high nanotube concentrations. The maximum nucleant effect was observed at low nanotubes concentration $(0.5 \%)$. Similar results were obtained by dynamic mechanical analysis, with an increase of the storage modulus by incorporating of low nanotubes contents. However, a further increase in the SWNTs content dramatically decreases the storage modulus. Raman spectroscopy and scanning electron microscopy were successfully applied to demonstrate that changes in the properties of the composite films, can be explained in terms of the structural variations in the composite.

Keywords: Carbon nanotubes, TPOs, Raman spectroscopy

\section{INTRODUCCION}

El descubrimiento de los nanotubos de carbono (SWNTs) y materiales de carbono nanoestructurados ha inspirado el estudio de numerosos científicos durante los últimos años (1-3). Su inusual alto módulo de Young y resistencia a tracción junto a su baja densidad ha promovido el desarrollo de estos materiales como refuerzos de matrices poliméricas (4). La utilización de los nanotubos de carbono como refuerzo de polímeros no solo se debe a sus excelentes propiedades intrínsecas sino además, a su buena dispersión, alineamiento y propiedades interfaciales (5). SWNTs se caracterizan por una única estructura atómica con una alta relación longitud/diámetro. En la literatura, se ha indicado la capacidad reforzante de los nanotubos de carbono, mostrando considerables aumentos de las propiedades mecánicas del composite (6).

Por su parte, el polipropileno es uno de los materiales poliméricos más versátiles y de mayor consumo, gracias a un excelente balance entre sus propiedades físicas y mecánicas y su fácil procesabilidad a un coste relativamente bajo (7-10). Sin embargo, presenta pobres propiedades a bajas temperaturas debido a su alta temperatura de transición vítrea y elevada cristalinidad. Así, uno de los procedimientos más utilizados a nivel industrial para mejorar la tenacidad del PP, es la de incorporar matrices elastoméricas. Entre los diferentes elastómeros, se ha demostrado que los terpolímeros de etileno-propileno-dieno (EPDM) son los más efectivos. Estas mezclas denominadas comúnmente como elastómeros termoplásticos poliolefínicos (TPOs), combinan las buenas características de procesado de los termoplásticos a elevadas temperaturas (11) con las excelentes propiedades físicas de los elastómeros convencionales a las temperaturas de servicio $(12,13)$. No obstante, la incorporación de la fase elastomérica reduce algunas propiedades del PP, como el modulo o la resistencia. Es de esperar, que la incorporación de agentes reforzantes como los SWNTs mejoren algunas de las propiedades de estos sistemas. Por todo ello, el objetivo del presente trabajo es analizar el efecto combinado de la incorporación de un elastómero y de los SWNTs sobre la morfología y propiedades del PP. Además, se pretende evaluar el efecto de la concentración de nanotubos sobre las propiedades físicas y mecánicas del nanocomposite.

\section{PARTE EXPERIMENTAL}

Se utilizaron un polipropileno (iPP), (PP030) suministrado por Solvay y un terpolímero de etileno-propileno-dieno (EPDM), (Buna EPT 6470P) proporcionado por Bayer. Los nanotubos de carbono han sido suministrados por Carbolex con una pureza del 70\%. El 30\% restante está compuesto de níquel y carbono amorfo.

Los nanocomposites se prepararon en un mezclador interno, in- 
corporando diferentes porcentajes de nanotubos $(0.25,0.5,0.75$ y $1 \%)$ a la mezcla de PP-EPDM (70-30) en fundido.

El análisis térmico se realizó en un calorímetro diferencial de barrido, DSC Mettler Toledo modelo 822. La cinética de cristalización se estudió en condiciones isotérmicas a $132{ }^{\circ} \mathrm{C}$ y dinámicas a una velocidad de enfriamiento de $10^{\circ} \mathrm{C} / \mathrm{min}$. El procedimiento usado se describió en un trabajo previo (14). Las propiedades mecanodinámicas se determinaron en un analizador mecanodinámico Metravib modelo Mark 03. El test se llevó a cabo en modo de tensión-compresión a una frecuencia de $5 \mathrm{~Hz}$, con un programa de temperatura de $-80^{\circ} \mathrm{C}$ a $40{ }^{\circ} \mathrm{C}$ y una velocidad de calentamiento de $2^{\circ} \mathrm{C} / \mathrm{min}$. Los espectros Raman se obtuvieron usando un Jobin Yvon micro-Raman LabRam con geometría backscattering. Se utilizó un láser de He-Ne de 632.8 $\mathrm{nm}(1.96 \mathrm{eV})$ como fuente de luz y filtros ópticos para ajustar el láser. La morfología de las muestras se analizó en un microscopio electrónico de barrido (SEM), modelo Philips XL30 ESSEM.

\section{RESULTADOS Y DISCUSION}

La Figura 1 muestra las curvas del grado de cristalización del PP, obtenidas por integración de los termogramas isotérmicos a $132{ }^{\circ} \mathrm{C}$, para todos los composites estudiados. Se observa que los nanotubos actúan como nucleantes, acelerando notablemente la cristalización del PP. No obstante, es interesante destacar que este efecto no es lineal con el contenido de SWNTs en el composite. Así, se aprecia una nucleación máxima a concentraciones de $0.5 \%$ de SWNTs, disminuyendo la velocidad de cristalización a concentraciones superiores, si bien, la velocidad es superior a la de las correspondientes mezclas sin SWNTs. Este comportamiento se aprecia fácilmente al analizar los tiempos medios de cristalización, $\tau_{1 / 2}$ indicados en la Tabla 1 . Se observa que $\tau_{1 / 2}$ del PP en la mezcla, disminuye al aumentar la concentración de nanotubos en el composite, mostrando un mínimo a concentraciones de $0.5 \%$ de SWNTs. Este particular comportamiento se puede explicar a través del balance de dos contribuciones opuestas. Por un lado, los resultados indican que los nanotubos favorecen la nucleación en la interfase matriz-nanotubos, mientras por otro lado, los mismos SWNTs a altos porcentajes $(>0.5 \%)$ impiden el crecimiento esferulítico de los cristales del PP. El análisis cinético se evaluó aplicando la ecuación de Avrami (15):

$$
X_{v}(t)=l-\exp \left(-K t^{n}\right)
$$

donde $n$ es el exponente de Avrami, $K_{n^{\prime}}$ es la constante cinética y $t$ es el tiempo de cristalización. Los valores de $K$ y $n$ se calculan mediante la representación del $\log \left(-\ln \left(1-X_{1}\right)\right)$ vs $\log (t)$ (Figura 2). Los resultados obtenidos se indican en la Tabla 1. En todos los casos, se obtuvieron valores fraccionados de $\mathrm{n}(2<\mathrm{n}<3)$, indicando una nucleación heterogénea seguida por un crecimiento cristalino esferulítico. Por su parte, los valores de la constante cinética de cristalización, $K_{n^{\prime}}$ confirman las conclusiones anteriores, demostrando que la velocidad de cristalización del PP aumenta en presencia de los SWNTs, mostrando un máximo a concentraciones de $0.5 \%$.

Tabla 1. Parámetros de la cinética de Cristalización de los mateRIALES ESTUDIADOS

\begin{tabular}{|l|c|c|c|c|c|c|c|}
\hline & $\left.\mathrm{T}_{\mathbf{c}}{ }^{\circ} \mathrm{C}\right)$ & $\left.\Delta \mathbf{H}_{\mathrm{c}} \mathrm{J} / \mathrm{g}\right)$ & $\mathbf{X}_{\mathbf{c}}(\%)$ & $\left.\mathbf{T}_{\mathrm{m}}{ }^{\circ} \mathrm{C}\right)$ & $\mathbf{n}$ & $\mathbf{K}_{\mathbf{n}}\left(\mathrm{min}^{-\mathrm{n}}\right)$ & $\tau_{1 / 2}(\mathrm{~min})$ \\
\hline PP-EPDM & 118.1 & -39.63 & 27.1 & 161.2 & 2.46 & $1.94^{*} 10^{-2}$ & 4.28 \\
\hline PP-EPDM-0.25\% SWNT & 121.7 & -64.44 & 44.8 & 163.4 & 2.46 & $5.24^{*} 10^{-2}$ & 2.82 \\
\hline PP-EPDM-0.5\% SWNT & 122.2 & -64.97 & 44.4 & 163.7 & 2.35 & $1.09^{*} 10^{-1}$ & 2.21 \\
\hline PP-EPDM-0.75\% SWNT & 121.7 & -65.13 & 44.9 & 163.5 & 2.21 & $1.34^{*} 10^{-1}$ & 2.33 \\
\hline PP-EPDM-1\% SWNT & 121.7 & -58.70 & 40.1 & 163.5 & 2.20 & $1.52^{*} 10^{-1}$ & 2.58 \\
\hline
\end{tabular}

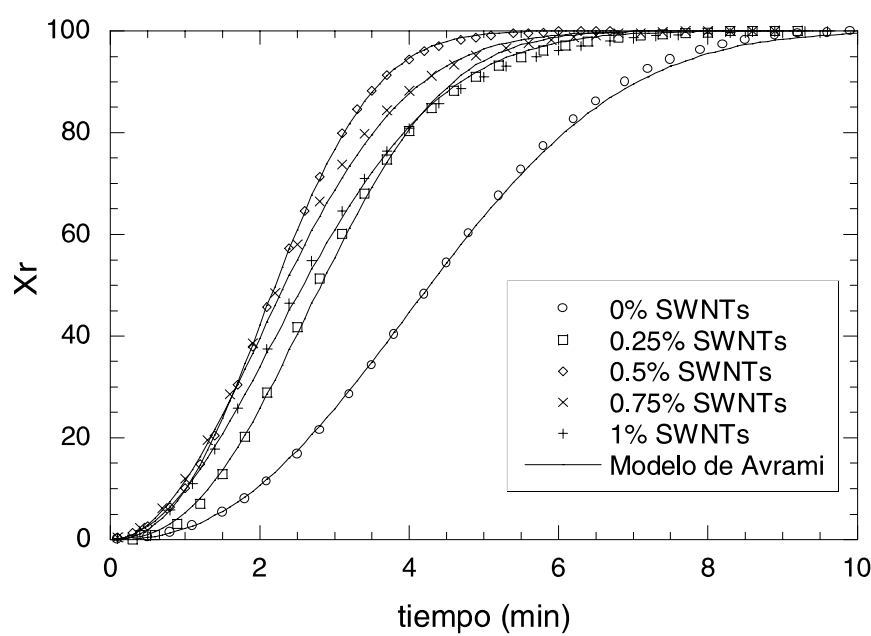

Figura 1. Curvas de cristalización isotérmicas de los nanocomposites PP-EPDM-SWNTs a $132{ }^{\circ} \mathrm{C}$.

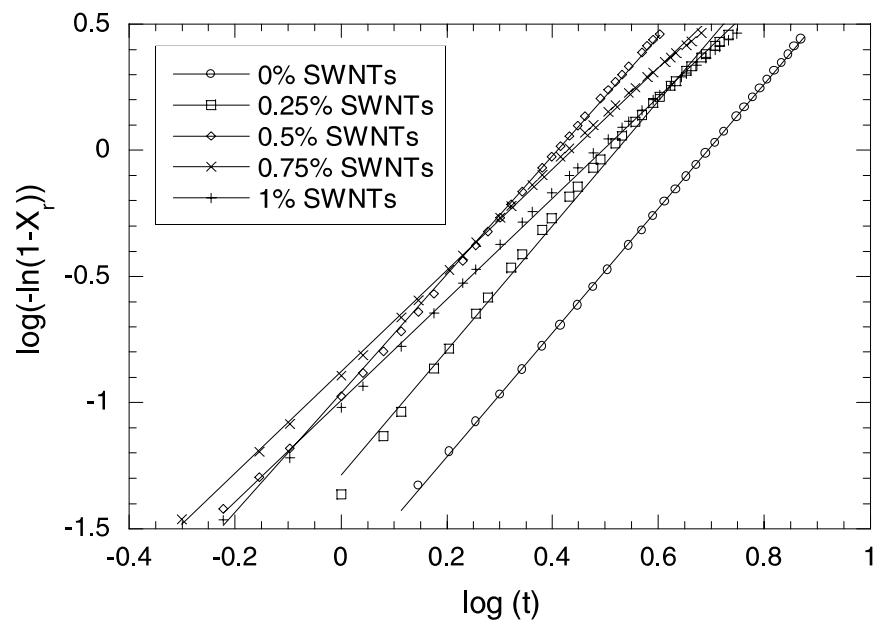

Figura 2. Representación de Avrami de la mezcla PP-EPDM y sus nanocomposites

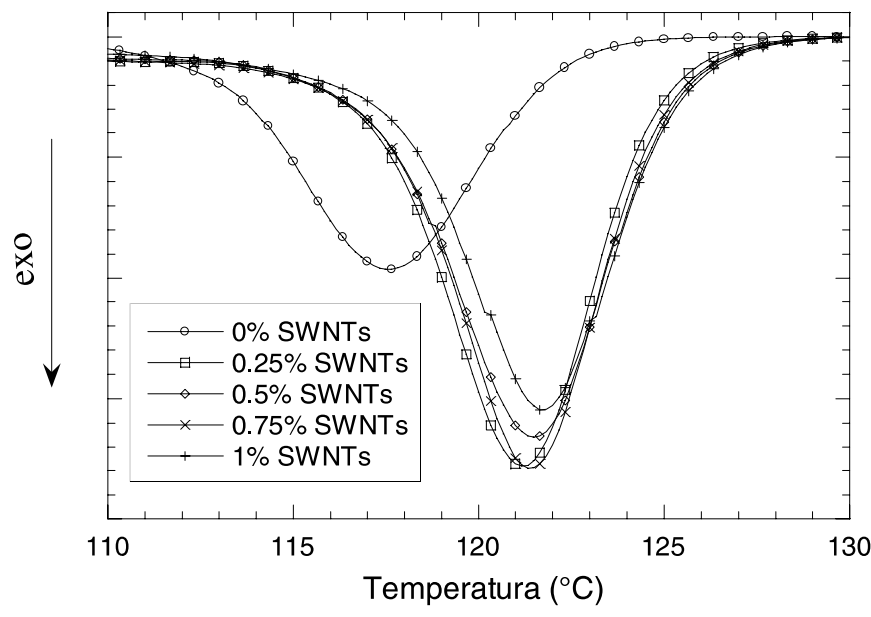

Figura 3. Curvas de cristalización dinámica de los nanocomposites PP-EPDM-SWNTs a $10^{\circ} \mathrm{C} / \mathrm{min}$

El efecto de los SWNTs sobre la cristalización del PP en mezclas PP-EPDM se estudió además, en condiciones dinámicas. La Figura 3 muestra las curvas de cristalización obtenidas a una velocidad de enfriamiento de $10{ }^{\circ} \mathrm{C} / \mathrm{min}$, para todos los materiales estudiados. La cristalización dinámica confirma los resultados previamente obtenidos, 
indicando el carácter positivo de los SWNTs sobre la cristalización del PP. Los valores medios del grado de cristalización $(X)$, temperatura de cristalización $(T)$, y temperaturas de fusión de las muestras cristalizadas $\left(T_{m}\right)$, se indican en la Tabla 2. De estos resultados, se deduce que la temperatura de cristalización aumenta en presencia de los SWNTs, si bien este efecto es más evidente para el composite reforzado con un 0.5 $\%$ de SWNTs. Por su parte, los SWNTs apenas varían la temperatura de fusión del PP.

La Figura 4 muestra las microfotografías para la mezcla PPEPDM sin SWNTs y sus nanocomposites con 0.5 y $1 \%$ de SWNTs. El nanocomposite con un $0.5 \%$ de SWNTs (Figura $4 \mathrm{~b}$ ) presenta una distribución uniforme y homogénea de los filamentos. Sin embargo, al aumentar el contenido de SWNTs en el nanocomposite (Figura 4c y d), se aprecia una mayor cantidad de agregados, disminuyendo la interacción carga-polímero.

La Figura 5 muestra el módulo de almacenamiento en función de la temperatura, para todos los materiales estudiados. En general, se aprecia que los nanotubos aumentan el módulo, observándose nuevamente un máximo a concentraciones de $0.5 \%$ de SWNTs en el nanocomposite. Posteriores aumentos de la concentración de SWNTs dan lugar a una considerable disminución del módulo, llegando incluso, en el caso del nanocomposite con 1\% de SWNTs a ser inferior al de la mezcla sin SWNTs. Estos resultados se explican en base al gran área superficial de los nanotubos de carbono. De este modo, a altas concentraciones de nanotubos en el composite (1\%), no existe polímero suficiente capaz de interaccionar con los nanotubos, de modo que aumentan las interacciones entre las cargas, formándose una mayor cantidad de agregados responsables del sensible descenso de las propiedades del material. Menard indicó resultados similares, explicándolos en función de las propias ondulaciones de los nanotubos de carbono (16).

Con objeto de determinar el efecto de los SWNTs sobre la microestructura de la mezcla PP-EPDM, los nanocomposites se caracterizaron mediante espectroscopía Raman. La Figura 6 muestra la existencia de un pico bien definido a bajas frecuencias $\left(100-200 \mathrm{~cm}^{-1}\right)$. Los espectros se analizaron mediante la búsqueda del número mínimo de frecuencias que ajustan la banda, obteniéndose tres picos a 145, 160 y $200 \mathrm{~cm}^{-1}$ asignados al modo breathing $\mathrm{A}_{1 \mathrm{~g}}$ de los nanotubos, cuya intensidad depende de la inversa del diámetro (17-20). La Figura 7 representa el desplazamiento de los picos en función de la concentración de SWNTs en el composite. Se deduce que los nanotubos desplazan la posición

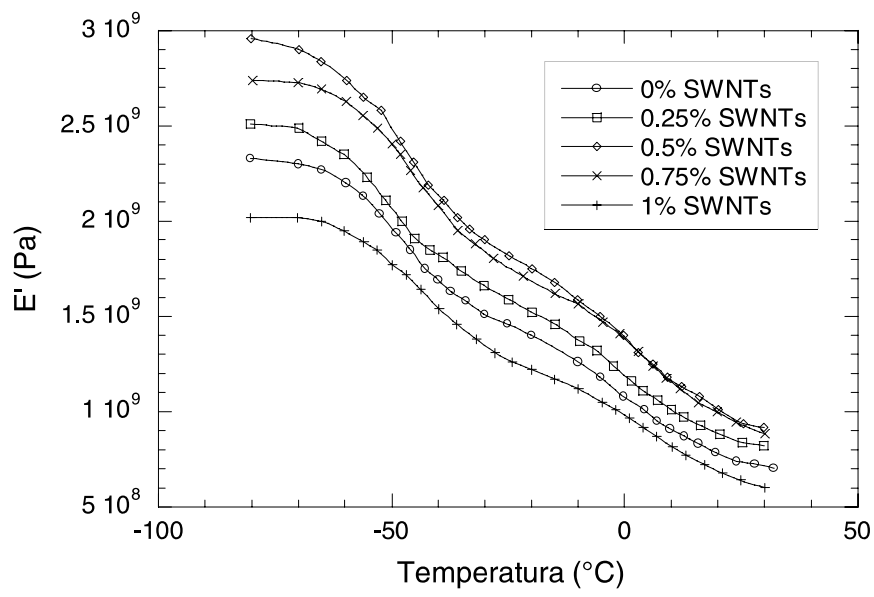

Figura 4. Imágenes SEM: a) PP-EPDM sin SWNTs, b) nanocomposite al $0.5 \%$ de SWNTs, c) nanocomposite al $1 \%$ de SWNTs y d) nanocomposite al $1 \%$ de SWNTs a mayores aumentos.
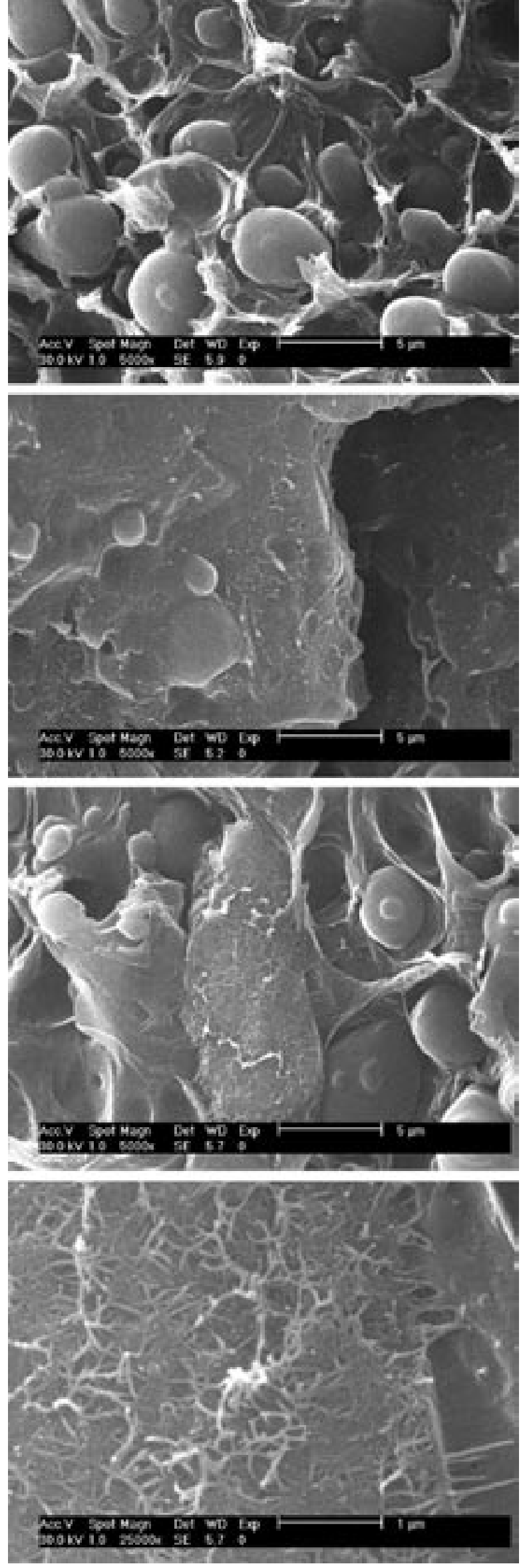

Figura 5. Módulo de almacenamiento de la mezcla PP-EPDM y sus nanocomposites. 


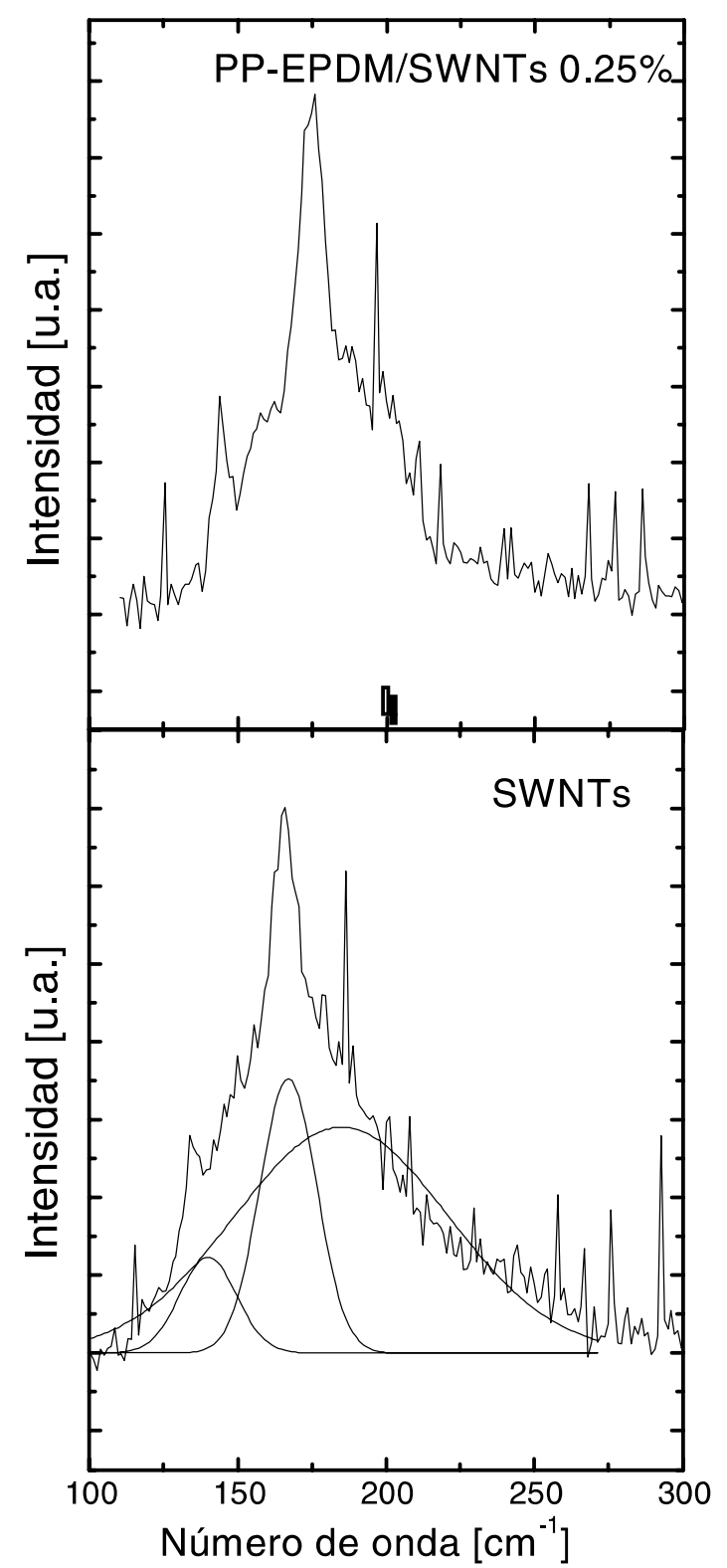

Figura 6. Espectros Raman de baja frecuencia de los nanotubos y del nanocomposite PP-EPDM-SWNT al $0.25 \%$.

del pico hacia mayores frecuencias, lo que se atribuye al efecto de la intercalación del polímero entre los haces del nanotubo. De hecho, el polímero ejerce una presión sobre los tubos individuales aumentando la frecuencia del modo breathing. Es interesante resaltar que este efecto es muy pronunciado con la incorporación de bajas concentraciones de SWNTs, mientras que apenas varía a concentraciones superiores. Esto indica que a bajas concentraciones, el polímero interacciona con los SWNTs, dando lugar a una apertura de los haces, de modo que los SWNTs se dispersan homogéneamente en la matriz polimérica. Sin embargo, a altas concentraciones de SWNTs, no existe polímero suficiente capaz de interaccionar con los SWNTs, por lo que aumentan las interacciones entre las cargas, formándose un mayor número de agregados. Por otro lado, el desplazamiento del pico depende del diámetro del nanotubo. Así, los picos a mayores frecuencias $(200 \mathrm{~cm}$ $\left.{ }^{1}\right)$, a los cuales les corresponden menores diámetros, apenas varían en presencia de los SWNTs.

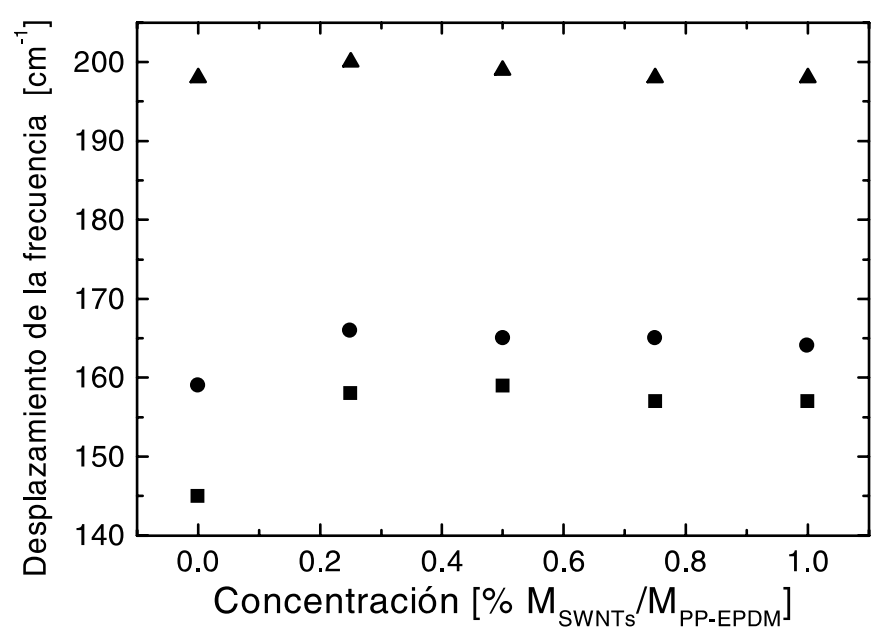

Figura 7. Variación de las bandas a bajas frecuencias en función de la concentración de SWNTs en el composite

\section{CONCLUSIONES}

En el presente trabajo, se han analizado las propiedades físicas y mecánicas de nanocomposites basados en elastómeros termoplásticos poliolefínicos reforzados con nanotubos de carbono. Los resultados demostraron que la incorporación de los nanotubos afectan a la microestructura y cristalinidad del PP, factores primordiales para interpretar la función de los nanotubos como refuerzos en matrices poliméricas. Se ha observado que estos efectos son más evidentes a concentraciones relativamente bajas $(0.5 \%)$ de SWNTs en el composite. Esto se explica en función del enorme área superficial de los nanotubos. Así, a bajas concentraciones, los SWNTs interaccionan mayoritariamente con el polímero, por lo que las interacciones carga-carga son pequeñas. De este modo, los haces de nanotubos se dispersan homogéneamente en la matriz polimérica. Sin embargo, al aumentar la concentración de SWNTs en el composite, no existe polímero suficiente capaz de interaccionar con los SWNTs, de modo que aumentan las interacciones entre las cadenas de nanotubos, formándose un mayor número de agregados. Esto ineludiblemente provoca una disminución de las propiedades del material.

\section{AGRADECIMIENTOS}

Los autores agradecen al Consejo Superior de Investigaciones Científicas (CSIC) y Consiglio Nationale delle Ricerche (CNR), su ayuda económica dentro del convenio de colaboración CSIC-CNR para el bienio (2001-2002). Dr. López Manchado agradece al Ministerio e Ciencia y Tecnología la concesión de un contrato dentro del Programa Ramón y Cajal.

\section{BIBLIOGRAFÍA}

1. S. Ijjima. "Helical microtubules of graphitic carbon", Nature, 354, 56-58 (1991).

2. W.A De Heer, A. Chatelain, D. Ugarte. “A carbon nanotube field-emissonelectron source", Science, 270, 1179-1180 (1995).

3. P.G. Collins, A. Zettl, H. Bando, A. Thess, R.E. Smalley, "Nanotube, nanodevice", Science, 278, 100-103 (1997).

4. R. Andrews, D. Jacques, M.A. Rao, T. Rantell, F. Derbyshire, Y. Chen, J. Chen, R.C. Haddon, "Nanotube composite carbon fibers", Appl. Phys. Lett. 75, 1329-1331 (1999).

5. O. Lourie, D.M. Cox, H.D. Wagner. "Buckling and collapse of embedded 
carbon nanotubes", Phys. Rev. Lett. 81, 1638-1641 (1998).

6. V.G. Hadjieva, M.N. Iliev, S. Arapalli, P. Nikolaev, B.S. Files. “Raman scattering test of single-wall carbon nanotube composites", App. Phys. Lett. 78, 3193-3195 (2001)

7. M. Alexandre, P. Dubois. “Polymer-layered silicate nanocomposites: preparation, properties and uses of a new class of materials", Mat. Sci. Eng. 28, $1-63(2000)$.

8. J. Jammak, S. Cheng, A. Zhang, E. Hsieh. “Isotacticity effect on crystallization and melting in polypropylene fractions: 3 . overall crystallization and melting", Polymer, 33, 728-735 (1992).

9. F.L. Bisbergen, B. DeLange, "Heterogeneous nucleation in the crystallization of plyolefins: Part 2. kinetics of crystallization of nucleated polypropylene", Polymer, 11, 309-332 (1970).

10. E. Martuscelli, M. Pracella, G.D. Volpe, P. Greco, “Morphology, crystallization and thermal behavior of isotactic polypropylenelow-density polyethylene blends", Makromol. Chem. 185, 1041-1061 (1984).

11. D. J. Synnott, D.F. Sheridan, E.G. Kontos, in "Thermoplastic elastomers from rubber-plastic blends", S.K. De, A.K. Bhowmick. Ellis Horwood (Eds.), New York, (1990)

12. D. Hoppner, J.H. Wendorff. "Investigations of the influence on the phase morphology of pp-epdm-blends on their mechanical properties", Colloid
Polym. Sci. 268, 500-512 (1990)

13. J. Karger-Kocsis, I. Csiku, "Skin-core morphology and failure of injectionmolded specimens of impact-modified polypropylene blends", Polym. Eng. Sci. 27, 241-253 (1987)

14. M.A. López-Manchado, J. Biagiotti, L. Torre, J.M. Kenny. “Polypropylene crystallization in an ethylene-propylene-diene rubber matrix", J. Therm. Anal. Cal. 61, 437-450 (2000)

15. M.J. Avrami, Kinetics of Phase Change. I. General Theory, J. Chem. Phys. 7, 1103-1112 (1939).

16. K. P. Menard, “Dynamic mechanical analysis: a practical introduction”, CRC Press, Boca Raton, (1999).

17. A.M. Rao, A. Jorio, M.A. Pimenta, M.S. Dantas, R. Saito, G. Dresselhaus, M.S Dresselhaus, "Polarized raman study of aligned multiwalled carbon nanotubes", Phys. Rev. Lett, 84, 1820-1823 (2000).

18. S.D. Brown, A. Jorio, G. Dresselhaus, M.S. Dresselhaus. "Observations of the d-band feature in the raman spectra of carbon nanotubes", Phys. Rev. B, 64, 73403-73406 (2000).

19. S. Bandow and S. Asaka, "Effect of growth temperature on the diameter distribution and chirality of single-wall carbon nanotubes", Phys. Rev. Lett., 80, 3779-3782 (1998).

20. M.N. Iliev, A.P. Litvinchuk, S. Arepalli, P. Nikolaev, C.D. Scott, “Fine structure of the low-frequency raman phonon bands of single-wall carbon nanotubes", Chem. Phys. Lett. 316, 217- 221 (2000).

Recibido: 1.2.03

Aceptado: 30.11 .03 